\title{
In vitro chemoresponse in metachronous pairs of gyneclologic cancers
}

\author{
Heather J Dalton ${ }^{1}$, James Fiorica ${ }^{2}$, Candace K McClure ${ }^{3}$, Rodney P Rocconi ${ }^{4}$, Fernando O Recio ${ }^{5}$, John L Levocchio ${ }^{6}$,
} Matthew O Burrell ${ }^{7}$ and Bradley J Monk ${ }^{8^{*}}$

\begin{abstract}
Background: While most gynecologic cancers respond to first-line cytotoxic chemotherapy, treatment of recurrent disease is frequently associated with acquired drug resistance. In order to find an in vitro surrogate of this clinical phenomenon, a tumor chemoresponse assay was studied.

Methods/Materials: Patients who had tissue submitted for repeated chemoresponse testing were identified through a retrospective search. Sixty-three patients met inclusion criteria (chemoresponse testing completed at primary diagnosis and upon recurrence of disease and assays completed $\geq 90$ days apart). The Wilcoxon signed-rank test was used to compare chemoresponse, represented as a response index (RI), between primary and recurrent measurements. In a secondary analysis, response was categorized and coded as Responsive $=3$, Intermediately Responsive $=2$ and Non-Responsive $=1$, and the paired $t$-test was used to compare chemoresponse between primary and recurrent measurement.

Results: Median time between primary and recurrent tumor testing was 309 days (IQR 208-422). Drugs tested included carboplatin, cisplatin, docetaxel, doxorubicin, gemcitabine, paclitaxel, topotecan, and combination carboplatin/gemcitabine and carboplatin/paclitaxel. There were no differences in chemoresponse between primary and recurrent measurement when chemoresponse was represented by RI scores; although a trend toward increased resistance to paclitaxel upon recurrence was noted. When chemoresponse was analyzed as a continuous variable corresponding to categorized response, a significant shift toward increased resistance to paclitaxel at recurrence, and a marginally significant trend toward increased resistance to carboplatin at recurrence, were observed.

Conclusions: We observed a trend toward increased chemoresistance at recurrence for paclitaxel, and a marginally significant trend toward increased chemoresistance to carboplatin, but no change in chemoresponsiveness between primary diagnosis and recurrence of disease for other common chemotherapy drugs, including common second-line agents such as doxorubicin, gemcitabine, and topotecan.
\end{abstract}

Keywords: Gynecologic cancer, Drug resistance, Recurrent ovarian, Chemotherapy, Cross-resistance

\section{Background}

It is estimated that in 2014, there were 21,980 new cases of ovarian cancer with 14,270 deaths [1]. The majority of patients present with advanced stage disease. While the majority of women will achieve complete clinical remission after cytoreductive surgery and platinum-based chemotherapy, approximately $30 \%$ of ovarian cancer patients do not have a complete response to front-line platinum-based

\footnotetext{
* Correspondence: bradley.monk@chw.edu

${ }^{8}$ University of Arizona Cancer Center, Creighton University School of Medicine at Dignity Health St. Joseph's Hospital and Medical Center, 500 W. Thomas Road, Suite 600, Phoenix, AZ 85013, USA

Full list of author information is available at the end of the article
}

treatment [2-6]. Furthermore, the majority of ovarian cancer patients recur and response rates to second-line treatments are substantially lower. This may be due, in part, to acquired drug resistance [7]. In the recurrent population, empiric-based chemotherapy is associated with response rates ranging from only 5 to $20 \%$ with limited progressionfree (PFS) and overall survival (OS) [8-11].

Since recurrence rates for ovarian cancer are high and the associated toxicity to chemotherapy can be significant, knowledge of potential tumor responses to chemotherapy a priori could be a useful tool in the selection of effective chemotherapy for patients with advanced or 
recurrent disease. Ineffective chemotherapy results in unnecessary toxicity and costs, delay of more effective treatment, and the potential development of acquired drug- and cross drug-resistance. An in vitro assay performed before therapy initiation to identify the drug(s) most likely to be effective for the individual patient would have clinical utility. Information provided by an in vitro assay, when integrated with clinical judgment, could lead to the identification of a potentially more effective treatment, thus eliminating toxicity due to ineffective treatments, avoiding a delay in the implementation of effective treatments, and potentially reducing treatment costs. Chemosensitivity and resistance assays are currently recognized in the National Comprehensive Cancer Network $\left(\mathrm{NCCN}^{\circ}\right)$ Clinical Practice Guidelines for Oncology for ovarian, fallopian tube, and peritoneal cancers $[12,13]$. ChemoFx is a live cell platform-based drug response marker that was developed to overcome the technical limitations of earlier generations of chemotherapy sensitivity and resistance assays and to determine chemosensitivity as well as chemoresistance [14]. Studies of this assay in ovarian cancer indicate that ChemoFx results are predictive of PFS [15] and OS [16].

There is currently limited information on whether chemosensitivity changes throughout the course of adjuvant chemotherapy administration and upon recurrence of disease. Therefore, the primary objective of this study is to determine whether in vitro tumor response differs in metachronous gynecologic cancer specimens collected from the same patient upon primary diagnosis and upon recurrence.

\section{Methods}

\section{Participants}

Inclusion criteria were as follows: (1) tumor samples were submitted to Precision Therapeutics, Inc. (Pittsburgh, Pa) for ChemoFx testing between August 2, 2006 and May 31, 2010; (2) chemoresponse testing was completed at primary diagnosis and upon recurrence of disease; and (3) assays were completed at least 90 days apart. A total of 63 participants met the inclusion criteria. Thirty-six of these patients were enrolled in the ChemoFx Physician Reported Outcomes (ChemoFx PRO) study. The research protocol was reviewed by the Copernicus Group Institutional Review Board, and they determined that this study qualifies as exempt research under 45 CFR $\$ 46.101$ (b) (4) before the initiation of the analysis. Existing de-identified (anonymized) clinical and pathological data were used to perform the analysis.

\section{ChemoFx}

Fresh tumor specimens preserved in McCoy's medium were received by the commercial laboratory, usually within 24 hours of removal. Tumor testing methods were reported previously [15]. Briefly, tumor specimens were mechanically disrupted to release and establish malignant epithelial cells as monolayer cultures. The tumor sample was then tested against a series of 10 serial dilutions of each drug or drug combination. The range of drug concentrations tested for each drug(s) was as follows: carboplatin $(1 \mu \mathrm{M}-500 \mu \mathrm{M})$, cisplatin $(0.2 \mu \mathrm{M}$ $100 \mu \mathrm{M})$, docetaxel $(0.1 \mathrm{nM}-25 \mathrm{nM})$, doxorubicin (2 nM$1 \mu \mathrm{M})$, etoposide ( $20 \mathrm{nM}-2 \mu \mathrm{M})$, gemcitabine ( $1 \mathrm{nM}-50$ $\mathrm{nM})$, ifosfamide $(0.2 \mu \mathrm{M}-100 \mu \mathrm{M})$, paclitaxel $(0.2 \mathrm{nM}-$ $0.1 \mu \mathrm{M})$, and topotecan $(0.4 \mathrm{nM}-0.2 \mu \mathrm{M})$, in addition to combination carboplatin/gemcitabine and carboplatin/paclitaxel. Combination drugs were tested as a 1:1 combination of each drug at each dose, for example, dose 1 of carboplatin-paclitaxel is dose $1(1 \mu \mathrm{M})$ of carboplatin plus dose $1(0.2 \mathrm{nM})$ of paclitaxel. After an incubation period of 72 hours, the cells were fixed with anhydrous ethanol (95\% fixing grade) and stained with 4'6-diamidino-2-phenylindole (DAPI), a fluorescent DNA stain for imaging the nucleus (Sigma-Aldrich Corp, St Louis, MO, USA). Cells that remained attached after staining were imaged and counted using automated cell microscopy and cell-counting software. The percentages of cells remaining after drug treatment were used to determine survival fraction $(\mathrm{SF}=$ average cell count dosex $_{\text {average cell count }}$ control $_{\text {, from which }}$ dose-response curves were plotted. Each dose-response curve was assigned a response index (RI) score ranging from 0 to 10. The RI score is a metric based on adjusted areas under the curve (aAUC); greater RI values represent greater sensitivity. Curves were smoothed using a logarithmic curve-fit tool (GraphPad Prism, LaJolla, CA, USA). Based on the aAUC score, in vitro tumor response was then categorized into three groups: responsive $(\mathrm{R})$, intermediately responsive (IR), or non-responsive (NR). The thresholds for these classifications were established based on the 25th and 75th aAUC percentiles. Drugs tested included carboplatin, cisplatin, docetaxel, doxorubicin, gemcitabine, paclitaxel, and topotecan, in addition to combination carboplatin/gemcitabine and carboplatin/ paclitaxel.

\section{Statistical methods}

The Wilcoxon signed rank test was used to compare chemoresponse (RI score) between primary and recurrent measurement. In commercial reports, tumors are categorized as R, IR or NR to chemotherapies; we therefore completed a second analysis with code corresponding to $R=3, I R=2$ and $N R=1$, and utilized a twotailed Wilcoxon signed rank test to compare chemoresponse between primary and recurrent measurement. Statistical significance was set at $\mathrm{p}<0.05$. All analyses were performed with SAS (version 9.2; SAS Institute, Cary, $\mathrm{NC}$ ). 


\section{Results}

The demographic characteristics of the 63 patients who met inclusion criteria are shown in Table 1 . Of the 63 pairs, 44 $(70 \%)$ were diagnosed with ovarian cancer, $6(10 \%)$ with peritoneal cancer, 4 (6\%) with fallopian tube cancer, and $9(14 \%)$ with uterine cancer. Mean age of patients was $59( \pm 11)$ years. The majority of cases exhibited poor tumor grade (grade 3 ) and FIGO Stage III. In the subset of patients with known treatment information (those enrolled in the ChemoFx PRO study), all were treated with a platinum/taxane combination for at least one cycle between in vitro chemoresponse testing of their metachronous tumors. Treatment information in this subsample is shown in Table 2.

Because specific individual chemotherapy agents or combinations of agents tested in the assay were selected by the treating physician, determination of chemoresponse was not possible for every drug in each patient. Overall, median time between primary and recurrent assay testing was 309 days (IQR = 208,422; minimum 91; maximum 680).

When examined using the RI score, no significant differences were observed in chemoresponse between primary and recurrent assay results (Table 3); however, a trend toward increased resistance was observed for the drug paclitaxel $(p=0.08)$. When chemoresponse was examined using the commercially reported categories, a significant shift toward chemoresistance were observed for the drug paclitaxel $(\mathrm{p}=0.04)$ and a marginally significant shift was observed for the drug carboplatin $(p=0.06)$ (Table 4). There were no statistically significant differences between primary and recurrent measurement in the remaining drugs tested.

Table 1 Characteristics of patients at diagnosis $(N=63)$

\begin{tabular}{lc}
\hline Age (years) & $59.8 \pm 11$ \\
Cancer Type & \\
Fallopian Tube & $4(6 \%)$ \\
Ovarian & $44(70 \%)$ \\
Peritoneal & $6(10 \%)$ \\
Uterine & $9(14 \%)$ \\
Tumor Grade & \\
G1 Well & $1(2 \%)$ \\
G2 Moderate & $6(10 \%)$ \\
G3 Poor & $24(38 \%)$ \\
G4 Undifferentiated & $10(16 \%)$ \\
Unknown & $22(35 \%)$ \\
FIGO Stage & \\
I & $4(6 \%)$ \\
II & $4(6 \%)$ \\
III & $47(75 \%)$ \\
IV & $7(11)$ \\
Unknown & $1(2 \%)$ \\
\hline
\end{tabular}

Table 2 Treatment administered to the subset of patients enrolled in the ChemoFx (Precision Therapeutics, Inc., Pittsburgh, PA) Physician Reported Outcomes Study between assays

\begin{tabular}{ll}
\hline & $\mathbf{N}=\mathbf{3 6}$ \\
\hline Bevacizumab/Carboplatin/Paclitaxel & $2(6 \%)$ \\
Carboplatin/Cisplatin/Paclitaxel & $7(19 \%)$ \\
Carboplatin/Gemcitabine/Paclitaxel & $1(3 \%)$ \\
Carboplatin/Paclitaxel & $2(6 \%)$ \\
Cisplatin/Docetaxel/Paclitaxel & $22(61 \%)$ \\
Cisplatin/Paclitaxel & $1(3 \%)$ \\
\hline
\end{tabular}

\section{Discussion}

Our results suggest that chemoresponsiveness does not change between primary diagnosis of disease and recurrence of disease for the majority of drugs in patients with gynecologic cancer who experienced recurrence within approximately one year. These results are consistent with a large analysis of 334 metachronous pairs of epithelial ovarian cancer specimens performed by Tewari et al. Their analysis failed to show a significant difference in the drug resistance profile of primary tumors and matched recurrences in the same patient [17]. These findings, in addition to ours, may be unexpected but not inexplicable. Furthermore, these results are consistent with observations of biomarker expression in metachronous pairs of epithelial ovarian cancer. No significant changes in the expression of MDR1, p53, or HER2 were noted between primary diagnosis and relapse of disease in 66 patients [18].

A possible explanation for continued chemosensitivity following exposure to chemotherapy, as observed in our primary analysis, has been offered by Tewari et al. They

Table 3 Chemoresponse assay results (expressed as a continuous variable (RI score)) for metachronous tumor pairs

\begin{tabular}{lcccc}
\hline & $\begin{array}{c}\text { Number } \\
\text { of pairs }\end{array}$ & $\begin{array}{c}\text { Primary median } \\
\text { (IQR) RI SCORE }\end{array}$ & $\begin{array}{c}\text { Recurrent median } \\
\text { (IQR) RI SCORE }\end{array}$ & $\mathbf{p}$ \\
\hline Carboplatin & 46 & $5.59(4.89,6.09)$ & $5.32(4.94,5.80)$ & 0.28 \\
Carboplatin/ & 35 & $5.71(5.16,6.30)$ & $5.90(5.40,6.36)$ & 0.33 \\
Gemcitabine & & & & \\
Carboplatin/ & 41 & $6.17(5.15,6.67)$ & $5.92(5.25,6.29)$ & 0.48 \\
Paclitaxel & & & & \\
Cisplatin & 42 & $5.40(4.66,5.77)$ & $5.38(4.67,5.68)$ & 0.80 \\
Docetaxel & 42 & $5.26(4.74,5.75)$ & $4.96(4.43,5.42)$ & 0.14 \\
Doxorubicin & 49 & $5.17(4.20,5.58)$ & $5.11(4.56,5.56)$ & 0.82 \\
Etoposide & 39 & $5.49(4.66,6.00)$ & $5.41(4.83,5.84)$ & 0.85 \\
Gemcitabine & 48 & $5.27(4.46,5.55)$ & $5.11(4.68,5.55)$ & 0.64 \\
Ifosfamide & 30 & $5.58(5.32,5.80)$ & $5.68(5.25,5.87)$ & 0.90 \\
Paclitaxel & 47 & $5.59(4.99,6.21)$ & $5.30(4.80,5.74)$ & 0.08 \\
Topotecan & 52 & $5.39(4.65,5.74)$ & $5.20(4.54,5.65)$ & 0.99 \\
\hline
\end{tabular}


Table 4 Chemoresponse assay results (expressed as a continuous variable corresponding to categorized results $(R=3, I R=2, N R=1))$ for metachronous tumor pairs

\begin{tabular}{lcccc}
\hline & $\begin{array}{c}\text { Number } \\
\text { of pairs }\end{array}$ & $\begin{array}{c}\text { Primary median } \\
\text { (IQR) RI SCORE }\end{array}$ & $\begin{array}{c}\text { Recurrent median } \\
\text { (IQR) RI SCORE }\end{array}$ & $\mathbf{p}$ \\
\hline Carboplatin & 46 & $2(1-3)$ & $1(1-2)$ & 0.06 \\
Carboplatin/ & 35 & $2(1-3)$ & $2(2-3)$ & 0.36 \\
Gemcitabine & & & $2(1-3)$ & 0.67 \\
$\begin{array}{l}\text { Carboplatin/ } \\
\text { Paclitaxel }\end{array}$ & 41 & $3(1-3)$ & & \\
Cisplatin & 42 & $2(1-2)$ & $2(1-2)$ & 1.00 \\
Docetaxel & 42 & $1(1-2)$ & $1(1-2)$ & 0.51 \\
Doxorubicin & 49 & $1(1-2)$ & $1(1-2)$ & 0.89 \\
Etoposide & 39 & $2(1-2)$ & $2(1-2)$ & 0.21 \\
Gemcitabine & 48 & $1(1-2)$ & $1(1-2)$ & 0.33 \\
Ifosfamide & 30 & $2(1-2)$ & $2(1-2)$ & 0.83 \\
Paclitaxel & 47 & $2(1-3)$ & $1(1-2)$ & 0.04 \\
Topotecan & 52 & $1.5(1-2)$ & $1(1-2)$ & 0.70 \\
\hline
\end{tabular}

postulate that after initial debulking surgery, patients may harbor residual tumor in poorly vascularized areas. Such tumors would be shielded from systemic chemotherapy, retain their initial drug responsiveness, and contribute to tumor regrowth and recurrence. Because the length of time between end of treatment and subsequent recurrence plays an important role in the development of drug resistance, [19] it is possible that if we had examined patients with longer time to recurrence, we may have observed greater shifts toward increased resistance.

A study conducted by Zajchowski, et al. examined differences in biomarker expression via immunohistochemical analysis in primary and recurrent ovarian cancer specimens over a much longer time interval. In the majority of patients examined, the interval between treatments was $>2$ years, and as high as $\approx 8$ year from the primary to last-received recurrent specimen. These patients received between 1-5 prior chemotherapies. Though differences were found in the expression of certain markers within each matched tumor pair, overall biomarker profiles for the matched patient specimens were very similar [20]. A study examining a larger patient population is needed to validate these findings.

A commonly held view is that chemoresponsiveness may change between primary diagnosis and recurrence of disease with intervening administration of chemotherapy. This may be due to the selection and clonal expansion of drug-resistant cells. Indeed, we observed a trend toward increased resistance to paclitaxel in our primary analysis, and a significant shift toward increased chemoresistance for paclitaxel, and a marginally significant shift toward increased chemoresistance for carboplatin, when examining change categorically. Although the treatment information was not included in the scope of this entire analysis, many of the patients in this study are assumed to have received platinum/taxane-based treatment as firstline chemotherapy. This assumption is supported by the treatment information garnered from the subset of patients enrolled in the ChemoFx PRO study. Exposure to these particular drugs may, in part, explain the more evident shift towards resistance observed with carboplatin and paclitaxel from primary to recurrent tumor (i.e., acquired drug resistance). Notably, there was no evidence for the development of cross-resistance; we did not observe an increase in resistance at recurrence for common second-line treatment agents, such as doxorubicin, gemcitabine, and topotecan.

The theory of acquired drug resistance may explain our results and those of a previous study of metachronous pairs conducted by Matsuo et al.; paclitaxel resistance was higher upon recurrence in a sample of 29 epithelial ovarian cancer patients treated with adjuvant platinum/taxane therapy. In addition, in a sample of 65 patients, recurrent surgery after initial cytoreduction was significantly associated with increased resistance to paclitaxel [19]. Similar increases in paclitaxel resistance in recurrent disease have been reported in other series [20,21]. Additional studies have shown limited, albeit, significant increases in carboplatin [22] and more commonly, paclitaxel, [22,23] chemoresistance throughout the course of disease in primary epithelial ovarian cancer, with no changes in any other cytotoxic agents examined. Clinically, it has been shown that while taxanes have activity as later-line agents in ovarian cancer, resistance emerges over time and this increased resistance is very likely due to intervening therapies [24].

Heterogeneity of drug response is a serious clinical problem encountered when administering chemotherapy. Chemoresponse assays may help the physician avoid administering certain chemotherapeutic agents when a patient's tumor is found to be resistant to those agents. Our results suggest that the chemosensitivity profiles provided by ChemoFx in the primary setting may be valuable for use in both the primary and recurrent setting. Since very few patients undergo a secondary surgery, the opportunity to use results from tissue collected at initial cytoreduction underscores the importance of our current findings. The primary setting may be the physician's only chance to collect a specimen and thus chemoresponse results in the primary setting may be critical to that patient's future treatment.

The limitations of this study are those inherent to retrospective studies, including potential selection biases and lack of blinding and control groups. We were also limited in statistical power by a small sample size and short time to recurrence. Additionally, the use of assay results to guide chemotherapy treatment may be a potential confounder.

\section{Conclusions}

In summary, we observed a trend toward increased chemoresistance at recurrence for the common first-line gynecologic cancer treatment agent paclitaxel, and a 
marginally significant trend toward increased chemoresistance at recurrence for the first-line gynecologic cancer treatment agent carboplatin, but no change in chemoresponsiveness between primary diagnosis and recurrence of disease for other common chemotherapy drugs, including common second-line agents such as doxorubicin, gemcitabine, and topotecan. Our results are consistent with clinical observations and established biologic concepts, suggesting that an in vitro assay may be a useful tool in both research and clinical practice. Drug resistance remains a major obstacle in cancer therapy. Further research is needed to better elucidate the mechanisms by which tumors acquire drug resistance and to define the role of chemoresistance assays in the treatment of gynecologic cancers.

\section{Competing interest}

Candace K. McClure was an employee of Precision Therapeutics, Inc during the time of this trial. Additionally, during the time of this trial James Fiorica was on the speaker's bureau for Precision Therapeutics, Inc. All other authors report no competing interest.

\section{Authors' Contributions}

HJD and BJM had the concept and design for the manuscript. All authors carried out the study and assisted with the collection and assembly of data. All authors assisted with the writing of the manuscript. All authors read and approved the final manuscript.

\section{Acknowledgement}

The authors wish to thank Daniele A. Sumner, BA for her assistance in editing this manuscript. The authors are solely responsible for the content of this manuscript.

\section{Sources of support}

This study was supported in part by Precision Therapeutics, Inc., Pittsburgh, PA.

\section{Author details}

1The University of Texas MD Anderson Cancer Center, Houston, TX, USA ${ }^{2}$ Sarasota Memorial Hospital, Sarasota, FL, USA. ${ }^{3}$ Precision Therapeutics, Inc, Pittsburgh, PA, USA. ${ }^{4}$ University of South Alabama Mitchell Cancer Institute, Mobile, AL, USA. ${ }^{5}$ South Florida Center for Gynecologic Oncology, Boca Raton, FL, USA. ${ }^{6}$ North Shore LIJ Health System/Biomedical Research Alliance of New York, Manhassett, NY, USA. ${ }^{7}$ Georgia Gynecologic Oncology, Atlanta, GA, USA. ${ }^{8}$ University of Arizona Cancer Center, Creighton University School of Medicine at Dignity Health St. Joseph's Hospital and Medical Center, 500 W. Thomas Road, Suite 600, Phoenix, AZ 85013, USA.

Received: 21 February 2014 Accepted: 22 April 2014

Published: 6 December 2014

\section{References}

1. Siegel R, Ma J, Zou Z, Jemal A: Cancer statistics, 2014. CA Cancer J Clin 2014, 64:9-29.

2. du Bois A, Luck HJ, Meier W, Adams HP, Mobus V, Costa S, Bauknecht T, Richter B, Warm M, Schroder W, Olbricht S, Nitz U, Jackisch C, Emons G, Wagner U, Kuhn W, Pfisterer J, Arbeitsgemeinschaft gynakologische Onkologie Ovarian Cancer Study Group: A randomized clinical trial of cisplatin/paclitaxel versus carboplatin/paclitaxel as first-line treatment of ovarian cancer. J Natl Cancer Inst 2003, 95:1320-1329.

3. du Bois A, Weber B, Rochon J, Meier W, Goupil A, Olbricht S, Barats JC, Kuhn W, Orfeuvre H, Wagner U, Richter B, Lueck HJ, Pfisterer J, Costa S, Schroeder W, Kimmig R, Pujade-Lauraine E, Arbeitsgemeinschaft Gynaekologische Onkologie; Ovarian Cancer Study Group; Groupe d'Investigateurs Nationaux pour l'Etude des Cancers Ovariens: Addition of epirubicin as a third drug to carboplatin-paclitaxel in first-line treatment of advanced ovarian cancer: a prospectively randomized gynecologic cancer intergroup trial by the Arbeitsgemeinschaft Gynaekologische Onkologie Ovarian Cancer
Study Group and the Groupe d'Investigateurs Nationaux pour l'Etude des Cancers Ovariens. J Clin Oncol 2006, 24:1127-1135.

4. Fruehauf JP: In vitro assay-assisted treatment selection for women with breast or ovarian cancer. Endocr Relat Cancer 2002, 9:171-182.

5. Neijt JP, Engelholm SA, Tuxen MK, Sorensen PG, Hansen M, Sessa C, de Swart CA, Hirsch FR, Lund B, van Houwelingen HC: Exploratory phase III study of paclitaxel and cisplatin versus paclitaxel and carboplatin in advanced ovarian cancer. J Clin Oncol 2000, 18:3084-3092.

6. Ozols RF, Bundy BN, Greer BE, Fowler JM, Clarke-Pearson D, Burger RA, Mannel RS, DeGeest K, Hartenbach EM, Baergen R, Gynecologic Oncology Group: Phase III trial of carboplatin and paclitaxel compared with cisplatin and paclitaxel in patients with optimally resected stage III ovarian cancer: a Gynecologic Oncology Group study. J Clin Oncol 2003, 21:3194-3200.

7. McGuire WP, Hoskins WJ, Brady MF, Kucera PR, Partridge EE, Look KY, Clarke-Pearson DL, Davidson M: Cyclophosphamide and cisplatin compared with paclitaxel and cisplatin in patients with stage III and stage IV ovarian cancer. N Engl J Med 1996, 334:1-6.

8. Bookman MA: Developmental chemotherapy and management of recurrent ovarian cancer. J Clin Oncol 2003, 21:149s-167s.

9. Cannistra SA: Is there a "best" choice of second-line agent in the treatment of recurrent, potentially platinum-sensitive ovarian cancer? J Clin Oncol 2002, 20:1158-1160.

10. Ozols RF: Recurrent ovarian cancer: evidence-based treatment. $J$ Clin Oncol 2002, 20:1161-1163.

11. Markman M, Bookman MA: Second-line treatment of ovarian cancer Oncologist 2000, 5:26-35.

12. Chemosensitivity/Resistance assay included as part of the NCCN principles of chemotherapy. [http://www.medicalnewstoday.com/releases/180642.php]

13. NCCN clinical guidelines in oncology: epithelial ovarian cancer/ fallopian tube cancer/primary peritoneal cancer, version 2.2011. [http://www.nccn. org/professionals/physician_gls/pdf/ovarian.pdf]

14. Brower SL, Fensterer JE, Bush JE: The ChemoFx assay: an ex vivo chemosensitivity and resistance assay for predicting patient response to cancer chemotherapy. Methods Mol Biol 2008, 414:57-78.

15. Gallion H, Christopherson WA, Coleman RL, DeMars L, Herzog T, Hosford S, Schellhas H, Wells A, Sevin BU: Progression-free interval in ovarian cancer and predictive value of an ex vivo chemoresponse assay. Int J Gynecol Cancer 2006, 16:194-201.

16. Herzog TJ, Krivak TC, Fader AN, Coleman RL: Chemosensitivity testing with ChemoFx and overall survival in primary ovarian cancer. Am J Obstet Gynecol 2010, 203:68.e1-6.

17. Tewari KS, Mehta RS, Burger RA, Yu IR, Kyshtoobayeva AS, Monk BJ, Manetta A, Berman ML, Disaia PJ, Fruehauf JP: Conservation of in vitro drug resistance patterns in epithelial ovarian carcinoma. Gynecol Oncol 2005, 98:360-368.

18. Tewari KS, Kyshtoobayeva AS, Mehta RS, Yu IR, Burger RA, DiSaia PJ, Fruehauf JP: Biomarker conservation in primary and metastatic epithelial ovarian cancer. Gynecol Oncol 2000, 78:130-136

19. Matsuo K, Eno ML, Im DD, Rosenshein NB: Chemotherapy time interval and development of platinum and taxane resistance in ovarian, fallopian, and peritoneal carcinomas. Arch Gynecol Obstet 2010, 281:325-328.

20. McAlpine JN, Eisenkop SM, Spirtos NM: Tumor heterogeneity in ovarian cancer as demonstrated by in vitro chemoresistance assays. Gynecol Oncol 2008, 110:360-364.

21. Matsuo K, Im DD, Rosenshein NB: Increased paclitaxel resistance in recurrent epithelial ovarian cancer: analysis of metachronous tumors. Int J Clin Oncol 2010, 15:325-327.

22. Pospiskova M, Spenerova M, Pilka R, Kudela M, Hajdúch M, Srámek V, Melichar B, Cwiertka K: Repeated chemosensitivity testing in patients with epithelial ovarian carcinoma. Eur J Gynaecol Oncol 2010, 31:295-298.

23. Geisler JP, Linnemeier GC, Thomas AJ, Manahan KJ: Extreme drug resistance is common after prior exposure to paclitaxel. Gynecol Oncol 2007, 106:538-540.

24. McCourt C, Dessie S, Bradley AM, Schwartz J, Brard L, Dizon DS: Is there a taxane-free interval that predicts response to taxanes as a later-line treatment of recurrent ovarian or primary peritoneal cancer? Int J Gynecol Cancer 2009, 19:343-347.

doi:10.1186/2053-6844-1-7

Cite this article as: Dalton et al:: In vitro chemoresponse in metachronous pairs of gyneclologic cancers. Gynecologic Oncology Research and Practice 2014 1:7. 